have done, Ye and colleagues focused on the design of the beam itself to reduce the density while maintaining the modulus. They developed a two-step approach to fabricate a carbon lattice structure composed of hollow nested tubes. The 3D-printed polymer nanolattices, which serve as the templates, are first coated with a nickel layer through electroless plating and then pyrolysed. The nickel layer serves as a catalyst to ensure sufficient pyrolysis of the polymer at a low temperature of $873 \mathrm{~K}$. The carbon atoms released from polymer decomposition dissolve into the nickel coating and precipitate on both the inner and outer surfaces of the tubular nickel coating to form carbon shells. Removal of the nickel layer leaves a hollow tube-in-tube sandwich structure, in which the inner and outer tubes are connected by radially aligned struts with diameters below $10 \mathrm{~nm}$ (Fig. 1b). Nanostruts are formed by carbon precipitating on the diffusion channels within the nickel coating. In addition to serving as a catalyst, the conformal nickel layer maintains the dimensions of the sacrificial polymer template during pyrolysis, thus preventing the significant volume shrinkage. Consequently, the density of fabricated nanoarchitected carbon can be as low as $6.4 \mathrm{mg} \mathrm{cm}^{-3}$. As shown in an Ashby map of Young's modulus versus the density of various porous carbon materials in Fig. $1 a^{2-10}$, nanoarchitected carbon with a tube-in-tube structure exhibits a modulus above $1 \mathrm{MPa}$ in the ultralight regime, which is ten times higher than those of all the other carbon porous materials with similar densities. The high modulus is attributed to its ordered and architected structure (Fig. 1b). Compared with most low-density carbon materials with stochastic structures (represented by carbon aerogel and carbon nanotube foam in Fig. 1d,e), nanoarchitected carbon (for example, tube-in-tube carbon and pyrolytic carbon nanolattice in Fig. $1 \mathrm{~b}, \mathrm{c})$ has a higher modulus/stiffness because of a higher connectivity and load transfer efficiency. Finite-element analyses reveal that in nanoarchitected carbon with a tube-in-tube structure, the external load is mainly borne by both the outer tube and the cylindrical sandwich panel consisting of nanostruts. This result indicates that nanostruts have an important role in efficiently coupling and coordinating the inner and outer tubes during deformation.

With the two-step approach, the space between the inner and outer tubes can be tuned through changing the nickel coating thickness. With increasing outer tube diameter (with thicker nickel coating), the modulus can be further increased with almost the same density. Furthermore, tube wall thicknesses and the size and distribution of nanostruts connecting the inner and outer tubes are also crucial for tailoring of the modulus. The controlling of tube wall thicknesses and the nanostruts' size and distribution relies on the microstructures of the nickel coating and the pyrolysis process catalysed by the nickel coating, which still remain a mystery and therefore require more in-depth explorations.

The work of Ye and colleagues provides a way to synthesize nanoarchitected materials with both an ultralow density and an ultrahigh modulus. Their modulus is higher than that of other carbon porous materials in the ultralight regime but is still two orders of magnitude lower than the theoretical limit (Fig. 1a). An emerging and open question is whether and how the modulus of porous materials can be improved to reach the theoretical limits for the ultralight regime. This will rely on the design of optimal architectures, which could be based on bioinspired principles and machine learning approaches, on the development of advanced fabrication techniques to control the feature size at the nanoscale and to further exploit size strengthening and flaw insensitivity, and on the selection of suitable constituent materials. Ultrahigh modulus/ stiffness in the ultralight regime is expected to be accompanied by ultrahigh strength and other unprecedented mechanical properties (such as remarkable recoverability, impact and fatigue resistances, and flaw tolerance), heralding exciting advances in porous materials.

\section{Yujia Wang (D) and Xiaoyan Li(D)}

Center for Advanced Mechanics and Materials, Applied Mechanics Laboratory, Department of Engineering Mechanics, Tsinghua University, Beijing, China.

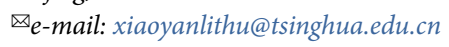

Published online: 25 October 2021 https://doi.org/10.1038/s41563-021-01131-y

References

1. Zheng, X. et al. Science 344, 1373-1377 (2014).

2. Ye, J. et al. Nat. Mater. https://doi.org/10.1038/s41563-02101125-w (2021).

3. Worsley, M. A. et al. Appl. Phys. Lett. 94, 073115 (2009).

4. Bauer, J., Schroer, A., Schwaiger, R. \& Kraft, O. Nat. Mater. 15 438-443 (2016)

5. Pekala, R. W., Alviso, C. T. \& LeMay, J. D. J. Non Cryst. Solids 125, 67-75 (1990).

6. Zhang, X. et al. J. Mater. Chem. 21, 6494-6497 (2011).

7. Qiu, L., Liu, J. Z., Chang, S. L., Wu, Y. \& Li, D. Nat. Commun. 3, 1241 (2012).

8. Barg, S. et al. Nat. Commun. 5, 4328 (2014).

9. Garcia-Tunon, E. et al. Adv. Mater. 27, 1688-1693 (2015)

10. Zhu, C. et al. Nat. Commun. 6, 6962 (2015).

Competing interests

The authors declare no competing interests.

\title{
Printing glass in the nano
}

\author{
A transparent suspension containing colloidal silica is used to additively manufacture three-dimensional structures \\ and photonic devices of silica glass at the nanoscale.
}

\section{Paolo Colombo and Giorgia Franchin}

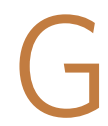
lass has always possessed a dual nature, oscillating between a long history of creativity and craftsmanship and compelling technological applications. It is an ancient material, but still widely used in modern industry due to its desirable mechanical properties, chemical inertness, thermal stability associated with a low expansion coefficient, electrical insulation and, most of all, excellent optical transparency. It finds wide applications in chemical and pharmaceutical processes, electronic and electrical technologies, high-temperature operations, light and laser technology, and optical system components. A simple example is its use in everyday products such as in smartphone screens and household equipment, another 


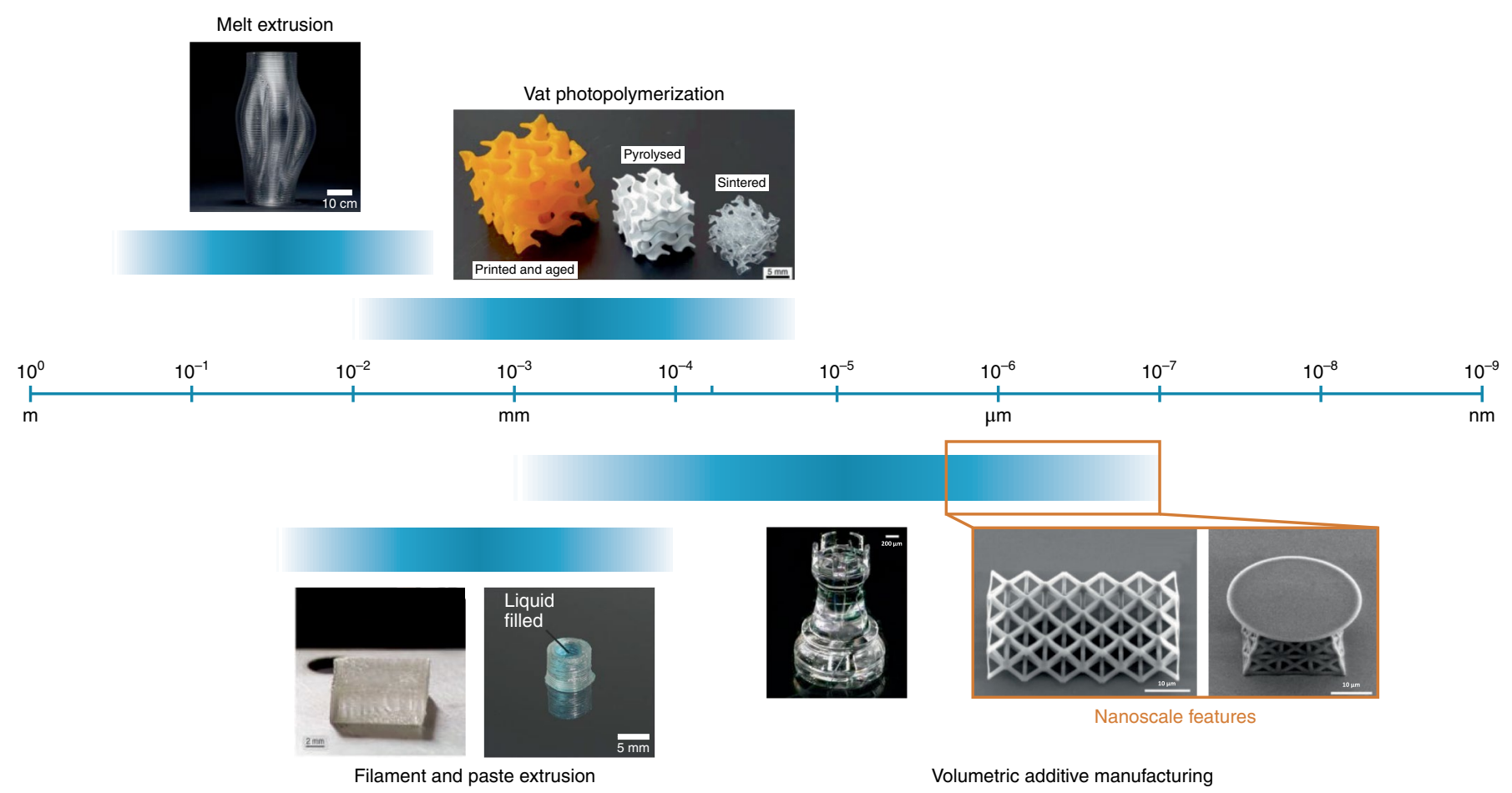

Fig. 1 | Techniques for additive manufacturing of glass at different length scales, from centimetric to nanometric features, with examples of glass components fabricated with each technique. Top left, light fixture lens. Top right, a gyroid-shaped object at different stages of the vat photopolymerization process. Bottom far left, an europium-doped phosphate glass sample. Bottom second left, cup containing dyed water, highlighting high-aspect-ratio features and merged printing lines. Bottom middle, a micro-rook with a height of $\sim 2 \mathrm{~mm}$. Bottom second right and far right, scanning electron microscopy images of an octet truss structure and a microtoroid optical resonator. Credit: top left, Liosdesign; top right, reproduced with permission from ref. ${ }^{7}$, Springer Nature Ltd. Bottom far left, reproduced with permission from ref. ${ }^{3}$, Elsevier; bottom second left, reproduced with permission from ref. ${ }^{4}$, Wiley; bottom middle, reproduced with permission from ref. ${ }^{8}$ Wiley; bottom second right and far right, reproduced with permission from ref. ${ }^{9}$, Springer Nature Ltd.

is its less obvious yet revolutionary use in communications as optical fibres.

The qualities of glass are strongly tied to its manufacturing techniques and each advance in technology can open new opportunities for its uses. Silica glass parts can be manufactured, for example by blowing, moulding and casting, from the melt at substantially higher temperatures than that forconventional soda-lime glass, but their short working window significantly limits the structures that can be obtained. Corning developed its multi-step Vycor process in the 1930s as a way to enable the production of (almost) pure silica components with a wider range of shapes using traditional fabrication technologies ${ }^{1}$, but challenges in obtaining high precision, small scale and complex architectures still remain. Additive manufacturing, which has developed rapidly and is becoming mature for plastics, enables the fabrication of parts with an almost unlimited variety of geometries, especially those not obtainable with traditional forming technologies. Much higher processing temperatures are required to form glass than to form plastics, therefore glass made a later entrance in the field. Different additive manufacturing techniques have been developed and demonstrated for the manufacturing of glass components, including melt extrusion ${ }^{2}$, filament extrusion ${ }^{3}$, paste extrusion ${ }^{4}$, stereolithography ${ }^{5}$, digital light processing ${ }^{6,7}$ and two-photon polymerization (2PP) ${ }^{8}$. However, the feature resolution for the complex components printed is still limited to the micrometre scale. Now, writing in Nature Materials, Xiewen Wen and colleagues ${ }^{9}$ report the fabrication of silica glass components with a resolution below $200 \mathrm{~nm}$, as shown in Fig. 1, based on the use of a highly transparent two-photon-polymerizable precursor containing colloidal silica nanoparticles.

The possibility of using photocurable suspensions based on colloidal silica ${ }^{5}$ or other photocurable systems ${ }^{6,7}$ was previously demonstrated in a vat photopolymerization layer-wise process. Technologies such as stereolithography ${ }^{5}$ and digital light processing ${ }^{6,7}$, which employ a selective and then subsequent exposure to a light source, can produce complex components but with features still limited in the range of 25 to $100 \mu \mathrm{m}$.
Instead of crosslinking a suspension layer-by-layer, by limiting the resolution to the thinness of the layer that can be produced, photopolymerization can be activated in a (small) volume of liquid; this is the case of $2 \mathrm{PP}$ technology $\mathrm{y}^{8}$, whose resolution is ideally limited only by the laser spot size. A volumetric additive manufacturing approach such as $2 \mathrm{PP}$ requires a very high transparency of the precursor to the radiation to initiate the reaction, which is not a typical feature that a suspension-based feedstock possesses. Transparency can be achieved by careful refractive index matching, and by controlling the amount of dispersed inorganic powders, which should, however, still be maintained above a certain level in order to avoid excessive shrinkage of the printed part and to allow for full densification upon sintering.

Wen and colleagues were able to achieve nanoscale resolution by using a commercially available polyethylene glycol (PEG)-functionalized colloidal silica with an $\sim 10 \mathrm{~nm}$ dimension. By matching the PEG functional groups with those of the photocurable polymers, they enhanced the 
suspension miscibility, obtaining a high loading of silica nanoparticles. Furthermore, the same refractive index possessed by the two feedstock components led to high transparency and limited scattering. This enabled the $3 \mathrm{D}$ printing of complex structures with sub-200-nm resolution (Fig. 1). The selection of the firing temperature $\left(1,300{ }^{\circ} \mathrm{C}\right.$ or $\left.1,100{ }^{\circ} \mathrm{C}\right)$ led to the production of either crystalline or amorphous silica. The researchers went one step further and employed their approach to fabricate microtoroid whispering gallery resonators with high quality factors, as well as active photonic devices produced by doping the suspension with photoluminescent rare-earth elements.

Two-photon polymerization has been demonstrated to be a suitable manufacturing approach for the production of specialized components, including metamaterials, photonic crystals, opto- and microfluidics, drug delivery devices, bioscaffolds for cell cultivation, freeform lenses and micro-optics. Indeed, the functionality of these structures is determined both by the architecture of the objects as well as by the material properties. The possibility of fabricating nanoscale three-dimensional parts with arbitrary shapes based on transparent silica glass thus greatly extends the range of applications enabled by $2 \mathrm{PP}$ technology. Although Wen and co-workers only presented a proof of concept, the exceptional sensitivity and the portability of whispering gallery resonators is of great interest for the production of biosensors and point-of-care diagnostic tests. A whispering gallery resonator made of erbium-doped silica has been applied for the detection of virus particles in air $^{10}$, a promising result with potential application for the prevention and control of epidemics such as the COVID-19 pandemic.

Despite the relevance of the work, challenges still remain in the accurate control of the shape resulting from the sintering process, as viscous flow leads to sagging and undesired deformations, as well as in obtaining full density and defect-free parts. Moreover, the use of a substrate, which is required by the $2 \mathrm{PP}$ technology, generates additional issues in terms of thermal expansion mismatch with the sample, as well as in limiting the type of components that can be fabricated. The use of a hybrid additive manufacturing approach, in which $2 \mathrm{PP}$ is coupled with digital light processing to obtain free-standing parts ${ }^{11}$, could provide a solution.

Paolo Colombo (DD 1,2凶 and Giorgia Franchin ${ }^{1}$ ${ }^{1}$ Department of Industrial Engineering, University of Padova, Padova, Italy. ${ }^{2}$ Department of Materials Science and Engineering, The Pennsylvania State University, University Park, PA, USA.

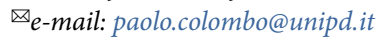

Published online: 25 October 2021

https://doi.org/10.1038/s41563-021-01137-6

References

1. Hood, H. P. et al. Treated borosilicate glass. US Patent 2,106,744 (1938).

2. Klein, J. et al. 3D Print. Addit. Manuf. 2, 92-105 (2015).

3. Zaki, R. M. et al. Mater. Design 194, 108957 (2020).

4. Nguyen, D. T. et al. Adv. Mater. 29, 1701181 (2017).

5. Kotz, F. et al. Nature 544, 337-339 (2017).

6. Cooperstein, I. et al. ACS Appl. Mater. Inter. 10, 18879-18885 (2018)

7. Moore, D. G. et al. Nat. Mater. 19, 212-217 (2020).

8. Kotz, F. et al. Adv. Mater. 33, 2006341 (2021).

9. Wen, X. et al. Nat. Mater. https://doi.org/10.1038/s41563-02101111-2 (2021).

10. He, L. N. et al. Nat. Nanotechnol. 6, 428-432 (2011).

11. Brigo, L. et al. Add. Manuf. 30, 100913 (2019).

Competing interests

The authors declare no competing interests.

\title{
Nano goes the distance
}

\author{
Centimetre-scale crack-free metal nanolattices are realized, enabling outstanding high tensile strength in \\ low-density materials.
}

\section{Andreas Stein and Nathan A. Mara}

T he ability to take metallic structures to greater heights with less weight was celebrated with great fanfare during the opening of the wrought-iron lattice tower built by Gustave Eiffel for the 1889 World's Fair. Eiffel's lattice approach to construction had a strong influence on architecture and has more recently inspired the design of low-density micro- and nanostructures, which can have attractive mechanical and physical properties that cannot be achieved in their bulk counterparts. This class of materials includes metamaterials used in photonics, smart pigments, display materials, sensors, energy storage and/or conversion materials, catalysts, bioactive materials and structural materials ${ }^{1,2}$. Their properties can be tuned by changing unit cell topology and size. Numerous phenomena emerge when the feature size is reduced to microand nanoscale, however, fabrication at these scales remains a challenge.

Additive manufacturing techniques are commonly used to generate lattice microstructures, and 3D printing using two-photon polymerization achieves the resolution needed for nanolattices. However, these sequential manufacturing techniques are relatively slow, and, for example, it would take several weeks to prepare a woodpile nanolattice structure at the centimetre length scale ${ }^{3}$. A faster, alternative approach is based on colloidal crystal templating, which uses arrays of uniform colloidal particles as a mould to prepare lattice structures with periodic porosity. The colloidal crystal is infiltrated with precursor material, which is then processed to convert the precursor to the target solid and to remove the particle template. The resulting, so-called inverse opals can be considered a class of metamaterials, but their applications are often limited by small sample dimensions. Typically, powders are obtained, or small chunks of thin-film materials are separated by frequent cracks. Although millimetre-sized, monolithic, inverse opals of some metals (for example, tungsten and tungsten-molybdenum alloys ${ }^{4}$ ) have been described, these contained defects and cracks throughout the structure. Now, writing in Nature Materials, Zhimin Jiang and James Pikul have been able to prepare self-standing, centimetre-scale inverse opal metallic nanolattices without cracks 